\title{
Performance of Hybrid Micro-Concentrator Module with Integrated Planar Tracking and Diffuse Light Collection
}

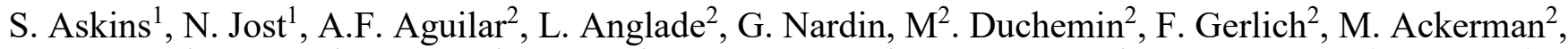 \\ L. Coulot ${ }^{2}$, D. Petri ${ }^{3}$, J. Levrat ${ }^{3}$, A. Faes ${ }^{3}$, J. Champliaud ${ }^{3}$, M. Despeisse ${ }^{3}$, C. Domínguez ${ }^{1}$, I. Anton ${ }^{1}$ \\ ${ }^{1}$ Instituto de Energía Solar, Universidad Politécnica de Madrid, 28040 Madrid, Spain \\ ${ }^{2}$ Insolight SA, Chemin de la Dent d'Oche 1A, CH-1024 Ecublens, Switzerland \\ ${ }^{3}$ CSEM PV-Center, Jaquet-Droz 1, CH-2002 Neuchâtel, Switzerland
}

\begin{abstract}
The Swiss start-up Insolight aims to be the first company to commercialize a high-efficiency III-V based low profile micro-CPV product that uses planar micro-tracking to eliminate the need for a tilting solar tracker, allowing rooftop mounting using typical flat-plate hardware, as well diffuse light capture using low cost $\mathrm{Si}$ solar cells which cover the area of the back plane not taken up by III-V solar cells. The IES-UPM has made an initial performance evaluation of a $0.1 \mathrm{~m} 2$ prototype. We show that the integrated planar tracking can reach $55^{\circ} \mathrm{AOI}$, show CSTC efficiency near to $30 \%$ for III-V output, and demonstrate the diffuse capture and planar tracking capability in a multi-week test campaign at our test site in Madrid.
\end{abstract}

Index Terms - integrated planar tracking, diffuse light collection, micro-concentrator photovoltaics.

\section{INTRODUCTION}

Concentrator Photovoltaics (CPV) has not lived up to the promise many saw for it a decade ago. While solar as a whole is experiencing exponential growth, reaching over $100 \mathrm{GW}$ of new capacity installed 2018, CPV capacity additions peaked at $120 \mathrm{MW} / \mathrm{yr}$ in 2012 and dwindled in the face of punishing price pressures from crystalline Silicon panels to $<5 \mathrm{MW} / \mathrm{yr}$ in recent years. [1]

The fact remains, however, that concentrators using III-V multi-junction (MJ) cells are, by a large margin, the most efficient solar technology on the planet, having reached $41.4 \%$ at the module level. [2] For this reason, many researchers are examining advances that will make CPV better suited for smaller systems on rooftops or other space constrained applications where value can be obtained from this efficiency advantage. These advances include:

- "Microscale" concentrators, or Micro-CPV, where-in the solar cells are $\leq 1 \mathrm{~mm}$ in size. This has myriad performance advantages (while introducing certain assembly complications). Importantly it means that CPV modules can have a form factor similar to flat plate (a few cm or less in thickness. [3]-[6]

- Planar microtracking: A frequently cited drawback to $\mathrm{CPV}$ is its requirement for a solar tracker, which limits feasibly of rooftop applications. By employing a second optical surface, it has been shown that high concentration optics can be designed which achieve focusing for high angles of incidence (AOI). [7]-[10]
- Diffuse capture: Another disadvantage faced by conventional $\mathrm{CPV}$ is that it only collects the direct beam resource. This produces a penalty of $10-20 \%$ during clear sky periods, and no power production at all in cloudy conditions, even if global irradiance is available. Low-cost Silicon solar cells can be combined with III-V cells under concentration to produce hybrid modules which capture direct light with high efficiency, and diffuse light with a lower efficiency. [11]-[14]

The above-mentioned advances are being carried out by universities, and to-date reported experimental results are for very small prototypes. The Swiss start-up Insolight has developed a module design that incorporates all three of these technologies, with a view to near-term industrialization, which would represent the first commercial solar panel with these features. They have moved from reporting $>36 \%$ efficiency on a small prototype in 2016 [15] to a $1 \mathrm{~kW}$ demo array installed at EPFL in 2018 , using $0.5 \mathrm{~m}^{2}$ panels with full integrated planar tracking (Fig. 1).

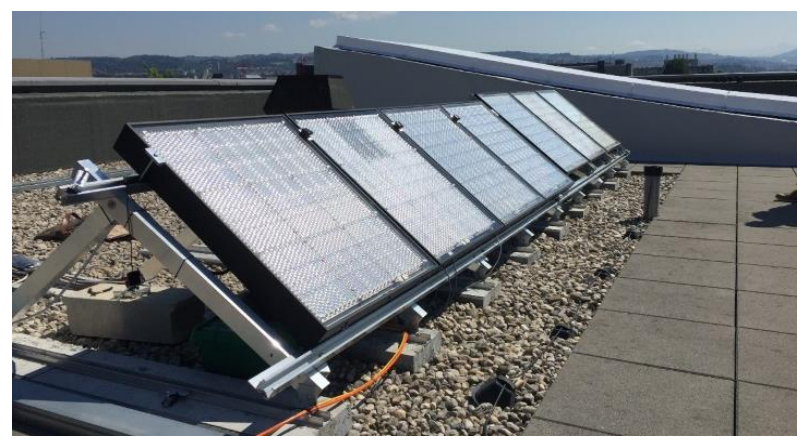

Fig. 1 A $1 \mathrm{~kW}$ demo array of Insolight's micro-CPV product installed at EPFL in Lausanne, Switzerland. A subset of these modules has been in operation for over 1 year. Observe that these highefficiency panels are installed south-facing and fixed-tilt using conventional flat-plate mounting hardware.

The Instituto de Energía Solar at the Madrid Technical University (IES-UPM) is collaborating with Insolight and since the end of 2018 has obtained two latest generation fully functional prototypes of a reduced scale $\left(0.1 \mathrm{~m}^{2}\right)$ version of their technology. In this work we present an initial performance evaluation, using both indoor and outdoor measurements. 
The Insolight technology employs a biconvex lens designed such that focusing is possible when the angle of incidence (AOI) approaches $60^{\circ}$, although the focal spot does travel as the sun moves, as shown in Fig. 2, and the entire back plane is translated to follow it, and maintain alignment. The back plane consists of an array of commercial triple junction microcells with approximately $42 \%$ efficiency combined with conventional 6" monocrystalline Silicon solar cells. The microcell size is $1 \mathrm{~mm}$ and the approximate geometric concentration ratio is $180 \mathrm{X}$. Because the optical elements are refractive, diffuse light which is not focused onto the III-V cells is instead collected by the Si cells, which cover the area not taken up by III-V cells. Voltages are not matched between III$\mathrm{V}$ and $\mathrm{Si}$ cells, so a four terminal output is provided.

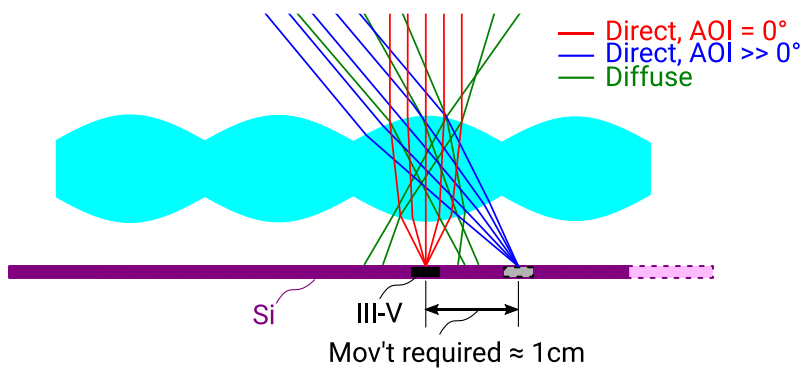

Fig. 2 Schematic of operation of the micro-planar tracking system and diffuse collection. Direct beam irradiance is collected by $1 \mathrm{~mm}$ III-V cells, while diffuse light is collected by the $\mathrm{Si}$ cell. For AOI not equal to $0^{\circ}$, the biconvex lens maintains a tight but translating focus. A simple mechanism causes the backplane to follow the focal point

\section{EXPERIMENTAL}

The module prototype can be seen in Fig. 3ab. The module size $\left(0.1 \mathrm{~m}^{2}\right)$ is reduced, but the construction follows the design intended for the final product: the module is fully enclosed, the features the actuation and control electronics for the integrated tracking system. In this prototype, the four 6" Si cells are in series, whereas the 572 micro-cells use a combination of series and parallel connection. Two modules have been measured, which are nominally identical (Modules A and B)

We will discuss the following measurement campaigns:

1. Outdoor power rating at normal incidents (module mounted on conventional solar tracker, Fig. 3) Module-A

2. Indoor power rating, and evaluation of angular performance of the planar tracking system, using a largearea collimated solar simulator. (Fig. 5) Module - $A$

3. Operational Testing of Integrating Tracking System: analysis of planar tracking and diffuse collection in a fixed tilt mounting configuration. Module- $B$

\footnotetext{
${ }^{1} \mathrm{CSOC}=$ Concentrator Standard Operating Conditions and CSTC = Concentrator Standard Test Conditions. See IEC 62670-1 \& -3. Due to ambient conditions, CSOC ambient temperature was not obtained.
}

We present some salient results from these measurement campaigns with a view to validating the integrated tracking system, and the diffuse capture.

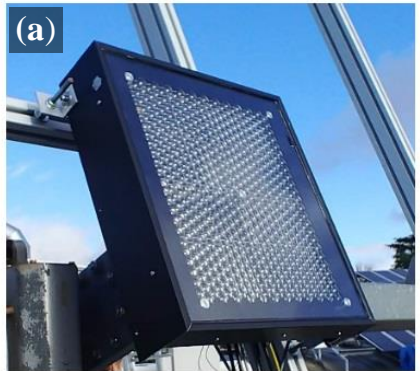

Voltage (Si) (V)
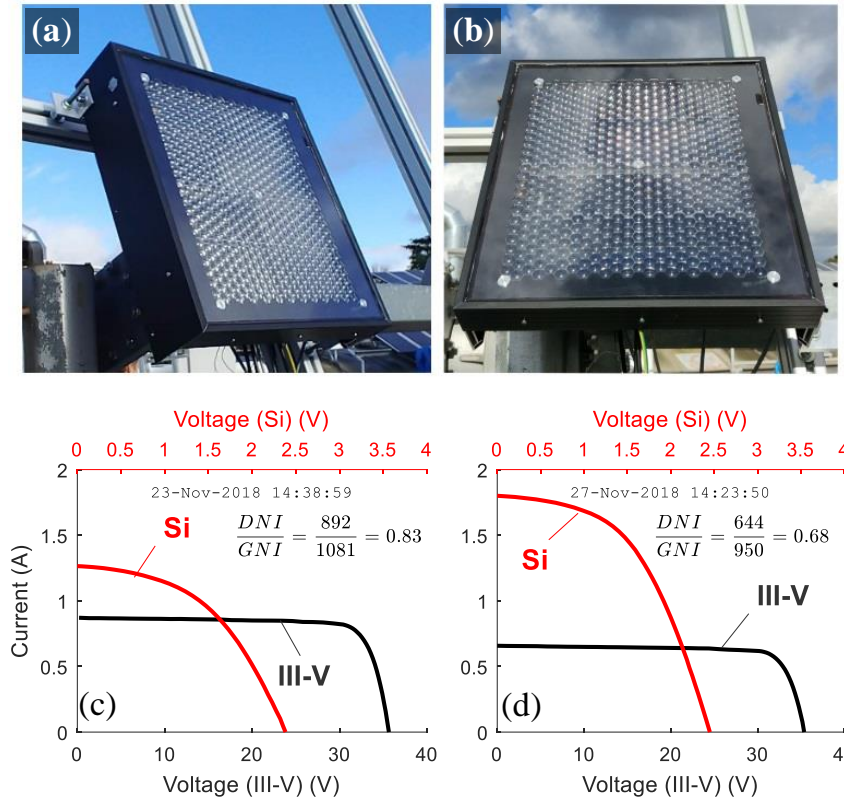

Voltage (Si) (V)

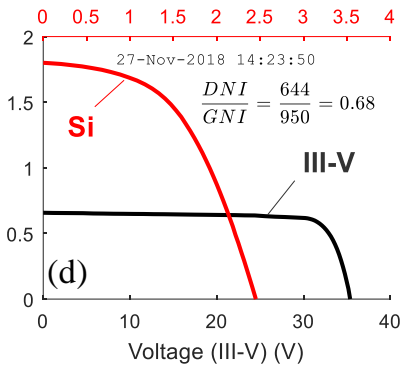

Fig. 3 (a,b) The four-terminal, $0.1 \mathrm{~m}^{2}$ Insolight micro-CPV module prototype tested by the IES-UPM mounted on a conventional CPV lab tracker for the outdoor power rating measurements. (c) Outdoor I-V curves of both outputs at CSOC irradiance and spectrum. (d) I-V curves under hazy conditions.

\section{A. Outdoor Power Rating}

The purpose of these initial outdoor measurements was to establish a base-line outdoor efficiency prior to extended testing using the onboard tracking mechanism. The Insolight module was mounted on a 2-axis lab tracker on the roof of the IES (Fig. 3 ). The internal tracking was configured for AOI $=0^{\circ}$. Both III$\mathrm{V}$ and $\mathrm{Si}$ outputs were attached to a an I-V tracer, and I-V curves were recorded every 30 s, with the module left in open circuit between curves. Simultaneously, the solar resource (DNI, GNI) and meteorological conditions ( $\left.\mathrm{V}_{\text {Wind }}, \mathrm{T}_{\text {Ambient }}\right)$ were monitored. Using a set of isotype cells of the same type as the MJ cells used in the module, the Spectral Matching Ratio (SMR) was also recorded. Approximately two weeks of measurement data was obtained in late 2018.

Averaging measurements for clear-sky conditions close to CSOC irradiance and spectrum ${ }^{1}$, we obtain average performance for DNI / GNI $=\left[892 \mathrm{~W} / \mathrm{m}^{2}\right] /\left[1081 \mathrm{~W} / \mathrm{m}^{2}\right]$ (83\%), Fig. 3c. The average $\mathrm{P}_{\mathrm{MP}}$ of the III-V was $25.3 \mathrm{~W}$, an efficiency (against DNI) of $\eta_{\text {III-V,DNI }}=27.4 \%$. In these lowdiffuse conditions the $\mathrm{Si}$ output adds an additional $1.6 \mathrm{~W}^{2}$, for a combined GNI-based efficiency of of $\eta_{\text {Total,GNI }}=24.5 \%$. In Fig. $3 \mathrm{~d}$, we show IV curves corresponding to hazy conditions with

2 In this prototype, a design limitation is producing high series resistance in the Si cells, causing low fill factors $(\mathrm{FF} \approx 60 \% @ 1$ sun) so we use current ratios to examine diffuse collection behavior. 
a DNI/GNI ratio of $68 \%$. In Fig. 4a,b we examine the behavior of the III-V and Si outputs over a 3-day sequence with varying conditions to understand the diffuse capture. In Fig. 4c, we observe that as the DNI/GNI ratio decreases (more diffuse light) the Si cell current is larger as a ratio with the III-V cell current, which is expected for diffuse capture.
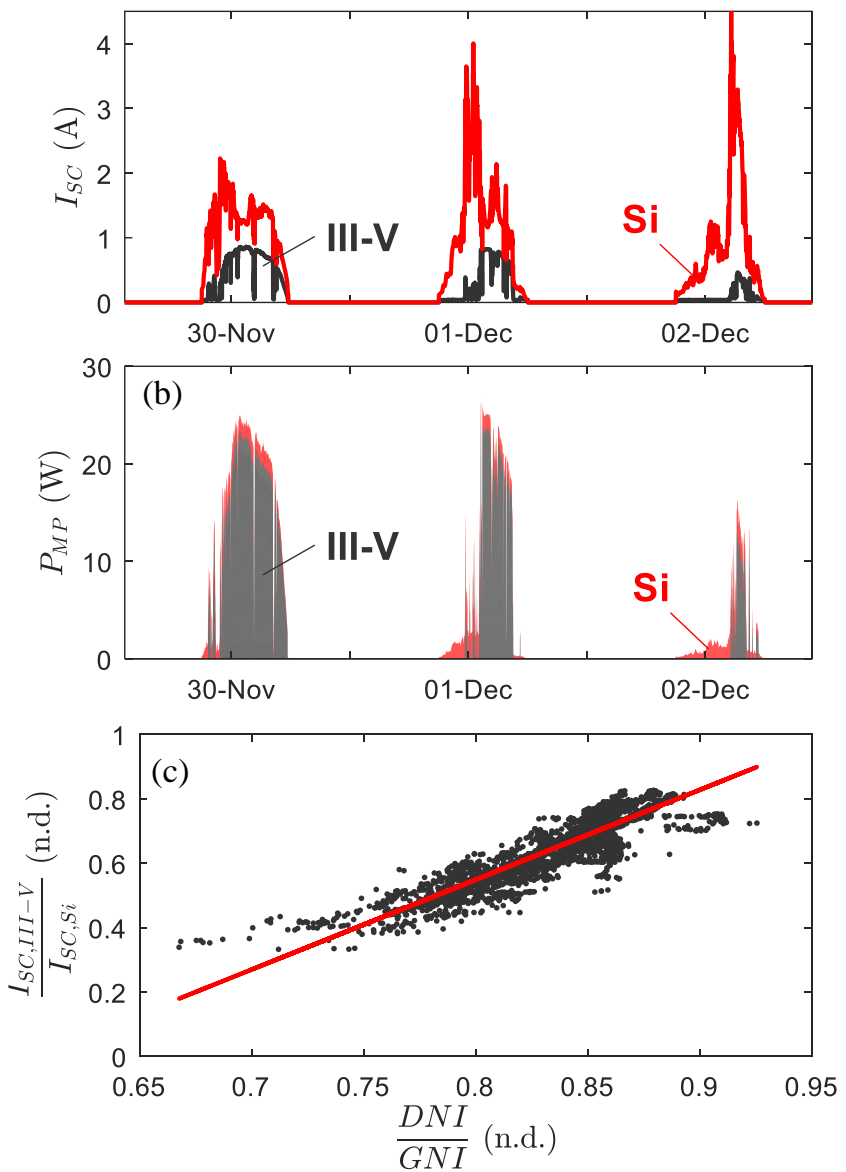

Fig. 4 Performance of diffuse capture for AOI $=0^{\circ}$ (on a solar tracker). (a) ISC of both outputs over the course of three days with varying conditions (mostly sunny on 30-Nov, partially cloudy on 1Dec, and mostly cloudy on 2-Dec.) The highest Isc,Si are observed when III-V current has been reduced due to clouds, and diffuse irradiance is higher. (b) Area chart showing power from both terminals: the Si output continues to produce power relatively constantly even as clouds cause III-V to drop to $0 \mathrm{~W}$. (c) The current ratio of the outputs as a function of DNI/GNI ratio over a period of approximately 2 weeks. See foot - note (2) on previous page.

\section{B. Indoor Measurements}

Indoor measurements were taken with the IES indoor large area collimated beam xenon flash tester $(2 \mathrm{~m}$ beam, $>1000$ $\mathrm{W} / \mathrm{m}^{2}, \pm 0.4^{\circ}$ collimation angle, AM1.5D matched by SMR). The setup is shown in Fig. 5a and Fig. 5b.

The goal of the indoor measurements was to establish the performance of the III-V $\mathrm{CSTC}^{1}$ rating, and to study the efficiency of the optics for AOI $\neq 0^{\circ}$, that is, with the module tilted with respect to the collimated beam of light produced by the simulator and with the micro-tracking used to align the cells to the focal spot produced by the biconvex lens. The CSTC results, both I-V curve and I-V parameters, are shown in Fig. 5c. These results are calibrated using the outdoor results presented in the previous section; that is the solar simulator was tuned such that the III-V output of the module produced the short circuit current observed outdoors in conditions of CSTC irradiance and spectrum, $0.96 \mathrm{~A}$ at $1000 \mathrm{~W} / \mathrm{m}^{2}$. A CSTC efficiency of $29 \%$ was measured for the on-axis condition.

The module was mounted on a rotating structure, Fig. 5b). and set at different values of AOI with respect to the collimated beam produced by the solar In Fig. 5d. we present the response of the normalized power and current versus AOI.
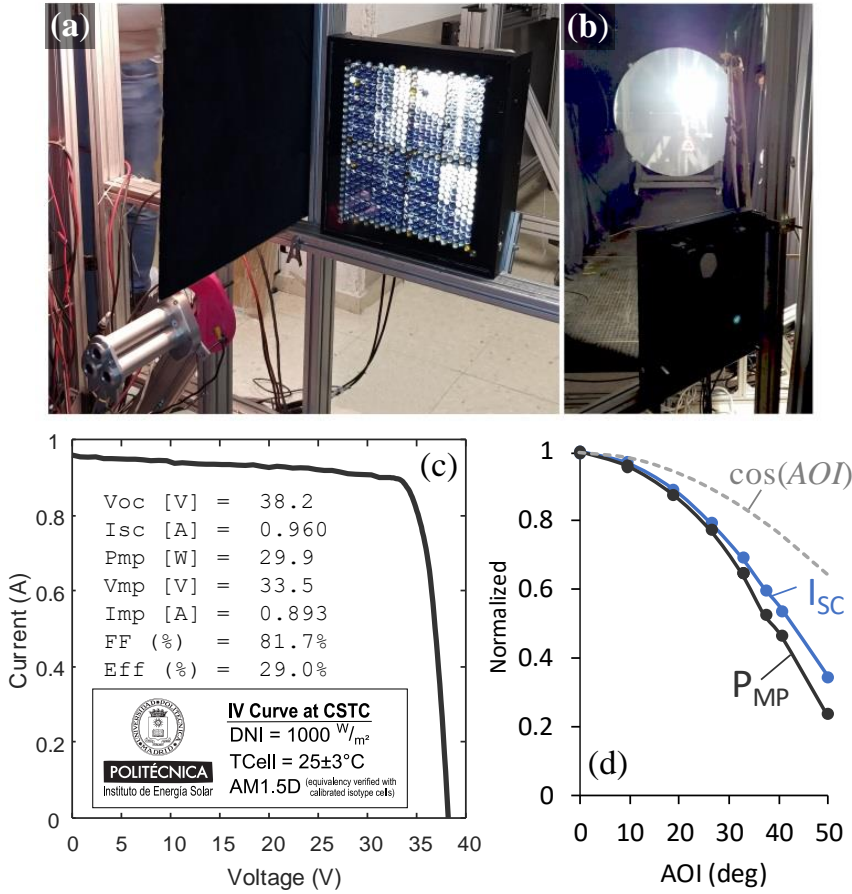

Fig. 5 Indoor measurement setup and results. (a) The module mounted perpendicular to the collimated beam from the mirror, along with isotype cells (lower left). (b) The measurement of the module at $\mathrm{AOI}=45^{\circ}$. The collimator can be seen in the background. (c) The I-V curve and parameters at $\mathrm{AOI}=0^{\circ}$. (d) Decrease in both current and power for AOI $>0^{\circ}$ (normalized to aligned values). IsC \& PMP fall $_{\text {M }}$ faster than cosine indicating lower optical efficiency for higher AOI

\section{Operational Testing - Setup}

For operational testing, the module was mounted outdoors on a solar tracker that was oriented to a fixed position and switched off for the duration of the tests. The slope angle was set to $30^{\circ}$ and the module was pointed due south. In Madrid, latitude $\approx$ $40^{\circ}$, the optimal tilt for yearly energy production approximately $\left(36^{\circ}\right)$.

A different module was used for these measurements, and this module was manufactured earlier than the module used for the measurements of, and showed lower performance, due to issues with internal alignment at assembly time that have since been resolved. 
Only the integrated tracking system was used to maintain focused light on the III-V solar cells. The module was provided by Insolight with an integrated microprocessor, firmware and actuators; no IES intervention was required for the module to begin tracking the sun once mounted other than for it to be activated according to Insolight instructions. The module uses proprietary hybrid (open/closed) control algorithm, the details of which are not known to the IES.

For the initial tests, both module outputs were placed into a short-circuit condition, and internal tracking was initiated. We chose to monitor only ISC for several reasons. Firstly, because it allows us to directly evaluate the amount of light arriving at the concentrating solar cells versus the diffuse capture solar cells under different internal tracking conditions, and secondly because the limitations of this specific module mean that the total power produced is not necessarily indicative of the potential of this technology. The module was additionally equipped with current and voltage measurement capabilities, which have been used for data recording in this test campaign. Simultaneously we have monitored the solar resource (DNI, GNI, GHI, and GII, Global inclined irradiance), as well as other ambient conditions, using the IES meteorological station.

Two weeks of data were gathered in the short circuitconditions. Afterwards, the module was measured using I-V curves, traced every $3 \mathrm{~min}$ on each output, with the module placed at MPP condition between curves, during one additional week.

\section{Operational Testing - Short Circuit Current Measurements}

In Fig. 7a, we show the current trace of the two module outputs, III-V and $\mathrm{Si}$, over the course of three days with increasingly clear skies. Above the figure, three example sky images are shown to provide a qualitative idea for the atmospheric conditions on each day. We make several observations. The module was observed to track during a period where the angle-of-incidence was between $\pm 55^{\circ}$. In this tracking period, the response of theIII-V cell is approximately cosine-shaped, with a maximum of around $0.8 \mathrm{~A}$ when the direct beam irradiance on the inclined plane (DII) was approximately $950 \mathrm{~W} / \mathrm{m}^{2}$. As the III-V cell reaches the maximum, the Si cell reaches a minimum in the middle of the day. This is related to the fact that the optical system concentrates the light to a smaller spot nearer to $\mathrm{AOI}=0^{\circ}$, for larger angles, some light is collected by the Si solar cell instead. It is noted that this means that the curve of Fig. 5d is pessimistic: some of the light lost by the III-V system is recovered.

Once the range limitation of the integrated tracking system is reached, the III-V solar cell array is commanded to its central position, and III-V output falls to zero. All light is collected by the $\mathrm{Si}$ system whose current more than doubles to nearly $3 \mathrm{~A}$.
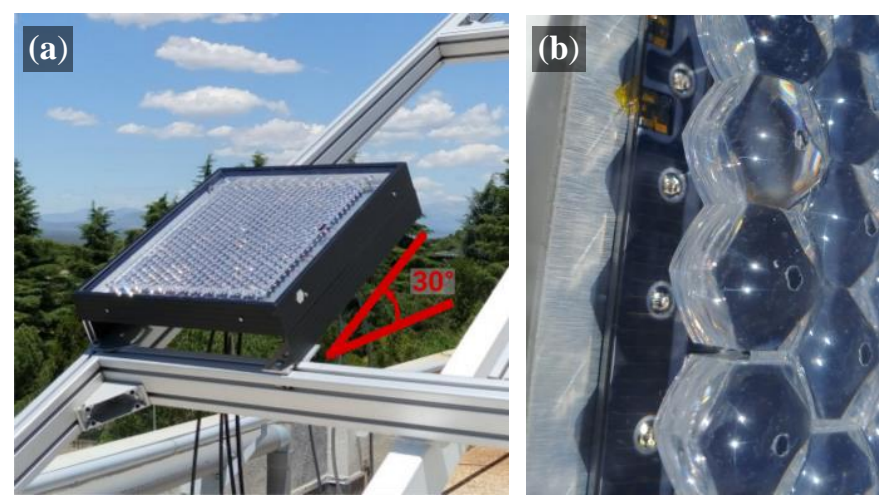

Fig. 6 Outdoor measurement setup for the operational testing of the integrating tracking system and diffuse light collection. (a) The module was mounted on fixed frame (a solar tracker that was instructed not to move). Inclination was set to $30^{\circ}$, and the module was pointed due south. (b) In this close-up view of the module tracking, we can see the biconvex lens, the spot of light focused on the III-V solar cells, and the silicon solar cell surrounding them.
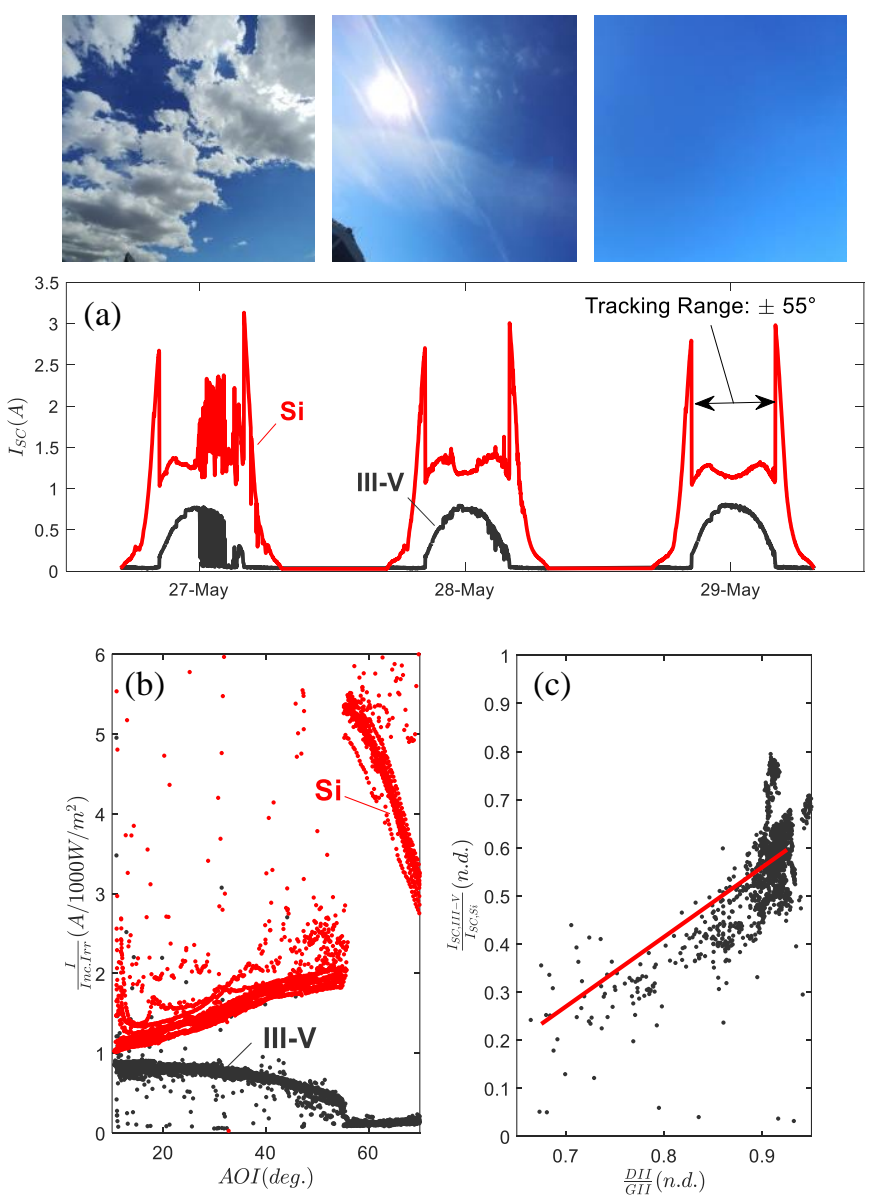

Fig. 7 Operational testing of integrated tracking system: (a) Shortcircuit current output of III-V and Si outputs over three days in May, with example sky images. (b) Examining the behavior of the integrated tracking system: normalized current vs. AOI. III-V current is normalized to DII and Si current is normalized to GII. (c) The graph of the ratio of III-V to current shows an expected positive trend with respect to the ratio of DII/GII. This data is shown only for AOI $<30^{\circ}$ in order to prevent effects of tracking from influencing the trend. 
For these high AOI, the size of the focal spot is increased, which is positive for the $\mathrm{Si}$ cell. We also observe that during the passing clouds on 27-may, the Si current is increased with greater diffuse light, as is expected.

In Fig.7b, we observe the irradiance-normalized-current output for recorded data vs. the AOI from the sun's position filtered for DNI/GNI > 0.7. Each output is normalized to an appropriate irradiance: the III-V output is normalized to DII, and the $\mathrm{Si}$ output is normalized to Global Inclined Irradiance (GII). In this figure, the trends discussed above are even more clear: in the case of the III-V response to AOI, we observe that at $\mathrm{AOI}=55^{\circ}$, the value normalized to the direct irradiance on the inclined plane has fallen to about half of its central value, confirming the data shown of Fig. 5d.

Finally, in Fig. 7c, we relate the ratio of the two currents to the ratio of DII/GII, as we did for $\mathrm{AOI}=0^{\circ}$ measurements in Fig. $4 \mathrm{c}$. These measurements were taken mostly on very clear days, as there is very little data for low values of DII/GII, but the observed trend is similar, although with a lower maximum due to angular effects. The integrated tracking does not appear to interfere with diffuse light collection.

\section{E. Operational Testing - Simple Model}

While it was more straight forward to measure the module outputs for two weeks in the short-circuit condition, we also would like to be able to make some observations on energy generation, so a simple model was used to use the measured current output to estimate the power output.

For the III-V output, we based this model on both the on-axis outdoor I-V curves (Section IIA), the indoor results, and the outdoor micro-tracked I-V curves taken after the short-circuit current measurements. We found that for on-axis I-V curves, the quantity $P_{M P P} / I_{S C}$ was quadratically related to DNI, as shown in Fig. 8a. It is assumed that this relationship encompasses both thermal effects and other effects of DNI on fill factor. The model is implemented piecewise to limit overestimation of the factor for DNI ranges which were not covered in the dataset. Also, it is known from Fig. 5d that we should expect a fill factor reduction for higher angle of incidence. Using the I-V curves traced in microtracking operation, we validated the indoor fill factor reduction due to AOI and then used the latter to fit a cubic polynomial (Fig. 8b. The combined model is:

$$
\begin{aligned}
& P_{M P, I I I V}=I_{S C, I I I V} \times V_{O C} F F_{I I I V}(D N I) \times F F_{R e d, I I I V}(A O I) \\
& \text { where: } \quad V_{O C} F F_{I I I V}=\left\{\begin{array}{cc}
A_{1}, & \text { if } D N I<550 \mathrm{w} / \mathrm{m}^{2} \\
A_{2}, & \text { if } D N I>1000 \mathrm{~W} / \mathrm{m}^{2} \\
A_{2}+B \cdot D N I+C \cdot D N I^{2}, & \text { otherwise }
\end{array}\right. \\
& F F_{\text {Red }, I I V}=D+E \cdot A O I+F \cdot A O I^{2}+G \cdot A O I^{3}
\end{aligned}
$$

In the case of the power output of the silicon solar cells, it was found that the quantity $P_{M P P} / I_{S C}$ showed little obvious relation to irradiance, but a linear dependence with the backplane temperature. (Fig. 8c) We also considered the possibility that the AOI would impact fill factor of the Si solar cells, specifically in the moment just before and after the tracking system activated in the morning and evening, when of concentrated sunlight was falling on these solar cells. However, looking at the variation of the normalized fill factor versus AOI during the portion of the operational testing in which I-V curves were collected, no increase in normalized fill factor was observed (Fig. 8d). The model for the Si output is summarized in Eqn. (3)-(4). Parameters for Eqn (1)-(4) are listed in Table I.

$$
P_{M P, S i}=I_{S C, S i} \times V_{O C} F F_{I I I V}\left(T_{B P}\right) \times F F_{R e d, S I}(A O I)
$$

where: $\quad V_{O C} F F_{S i}=H+I \cdot T_{B P}$ and $F F_{R e d, S I}=J=1$

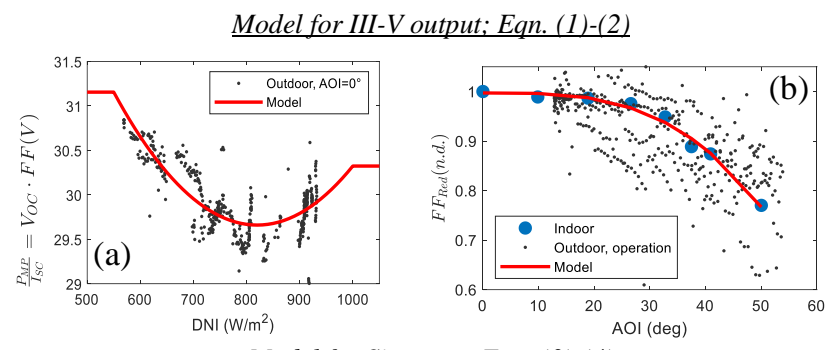

Model for Si output; Eqn. (3)-(4)

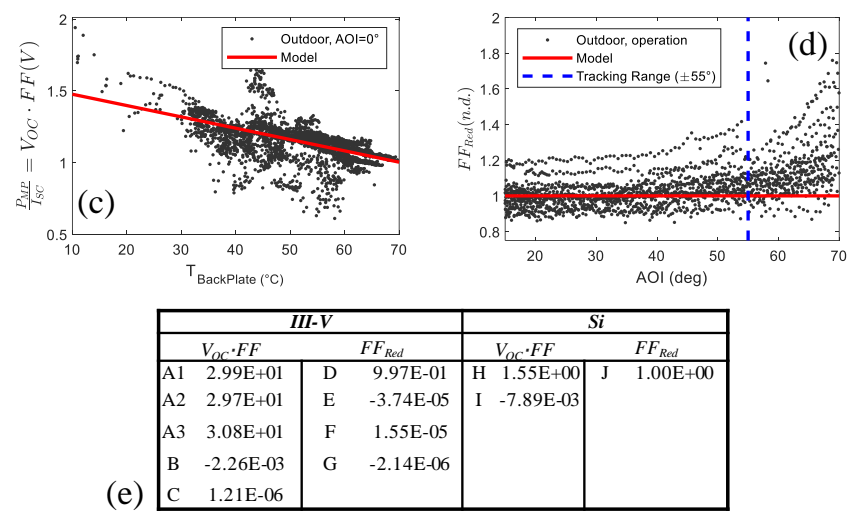

Fig. 8 (a) Quantity of $V_{O C} \times F F$ (ratio of $P_{M P}$ to $I_{S C}$ ) for the III-V output as a function of DNI. (b) Normalized $F F$ as a function of $A O I$ for the III-V output. Model is based on indoor data and validated to outdoor data collected in operation with I-V curve collection. (c) Quantity of $V_{O C} \times F F$ for the $\mathrm{Si}$ output as a function of module backplane temperature $\left(T_{B P}\right)$. (d) Normalized $F F$ as a function of AOI for the $\mathrm{Si}$ ouput. $F F$ reduction was expected to occur as concentrated light fell on $\mathrm{Si}$ cells for $\mathrm{AOI}>55^{\circ}$ (when microtracking ceased) However, this affect was not observed and no AOI dependence was included. (e) Coefficients for models of Eqn. (1)-(4).

Using these models, the two weeks of collected short circuit current values could be converted into estimated electrical power output for both cell types, as shown in Fig. 9a. Using these estimated power values, which had a 1-minute resolution, trapezoidal integration was used to calculate daily electrical generation for both the direct and diffuse outputs of the module over the course of 12 days in late May and early June. (Fig. 9b). 
(a)
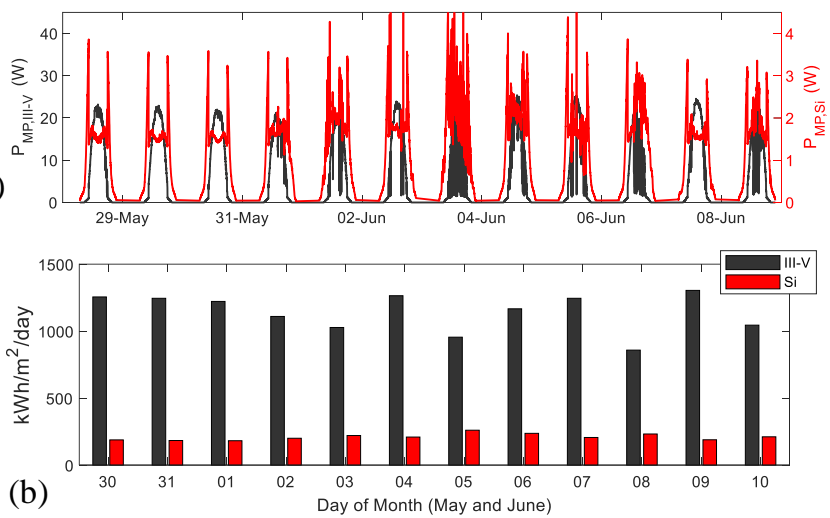

(c)

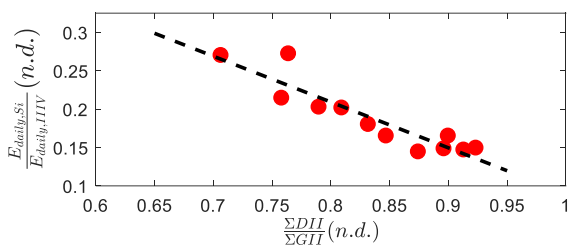

Fig. 9 (a) Estimated power output produced by combining the measured Isc over time (Section II.D) with the simple model of Section II.C. 12 days of data is shown. (b) Daily estimated electricity generation produced by integrating the data of (a) (d). Effect of the ratio of direct inclined (in-plane) irradiance (DII) and the global inclined irradiance (GII) on the energy produced by the Si cells as a proportion of energy produced by the III-V cells.

In addition, we calculated direct and global irradiances on the inclined plane of the module (DII and GII, respectively). It is shown in Fig. 9c that the amount of additional energy collected by the diffuse capture system, as a proportion of the energy generated by the micro-CPV system, varies linearly with the ratio of DII to GII, as is expected. For high diffuse days $(\mathrm{DII} / \mathrm{GII}<0.7)$ this "boost" from the Si cells can exceed $25 \%$.

\section{CONCLUSIONS}

In this work, we present an initial performance evaluation of a $0.1 \mathrm{~m}^{2}$ Insolight hybrid PV module prototype that shows nearly 30\% CSTC efficiency for direct capture. Although engineering tasks remain to optimize the technology, we have validated both its diffuse capture and planar tracking capabilities. Previous experimental characterizations on similar technologies has been conducted on smaller lab-scale prototypes, but this is the first module of its kind under commercial development, with near-term plans for industrialization. Long term measurements are ongoing in order to fully characterize this promising technology for high-density solar electricity production in space-constrained applications.

\section{ACKNOWLEDGMENTS}

These measurements were carried out with financing from the European Commission as part of the GRECO open science initiative and the Eurostars program. All data collected for these measurements has been made openly available at doi.org/10.5281/zenodo.3346823. The author's also acknowledge support of Innosuisse and would like to thank J. Brossard, N. Badel and G. Christmann of CSEM for support in preparing samples of the silicon solar cells.

\section{REFERENCES}

[1] D. S. Philipps and W. Warmuth, "Fraunhofer ISE Photovoltaics Report," 27-Aug-2018. [Online]. Available: www.webcitation.org/75bANcaZa.

[2] Karin Schneider and Gerald Siefer, "Concentrator Photovoltaics Shows Peak Performance - Module Efficiency Reaches $41.4 \%$ (Press Release)," 22-Nov-2018. [Online]. Available: www.webcitation.org/75XPiXcvF.

[3] G. N. Nielson et al., "Leveraging scale effects to create nextgeneration photovoltaic systems through micro- and nanotechnologies," in Micro- and Nanotechnology Sensors, Systems, and Applications IV, 2012, vol. 8373, p. 837317.

[4] S. Paap et al., "Cost analysis of flat-plate concentrators employing microscale photovoltaic cells for high energy per unit area applications," in Photovoltaic Specialist Conference (PVSC), 2014 IEEE 40th, 2014, pp. 2926d-2929.

[5] B. H. Jared et al., "Micro-concentrators for a microsystemsenabled photovoltaic system," Opt. Express, vol. 22, no. S2, p. A521, Mar. 2014.

[6] César Domínguez, et al., "A review of the promises and challenges of micro-concentrator photovoltaics," ,CPV-13, Ottawa, 2017.

[7] J. M. Hallas, et al., "Two-axis solar tracking accomplished through small lateral translations," Appl. Opt., vol. 51, no. 25, pp. 6117-6124, 2012.

[8] K. A. Baker, J. H. Karp, E. J. Tremblay, J. M. Hallas, and J. E. Ford, "Reactive self-tracking solar concentrators: concept, design, and initial materials characterization," Appl. Opt., vol. 51, no. 8, pp. 1086-1094, 2012.

[9] H. Apostoleris, M. Stefancich, and M. Chiesa, "Trackingintegrated systems for concentrating photovoltaics," Nat. Energy, vol. 1, no. 4, p. 16018, Apr. 2016.

[10] J. S. Price et al., "High-concentration planar microtracking photovoltaic system exceeding $30 \%$ efficiency," Nat. Energy, vol. 2, p. 17113, Jul. 2017.

[11] K.-T. Lee et al., "Concentrator photovoltaic module architectures with capabilities for capture and conversion of full global solar radiation," Proc. Natl. Acad. Sci. U. S. A., vol. 113, no. 51, pp. E8210-E8218, Dec. 2016.

[12] J. F. Martinez, M. Steiner, M. Wiesenfarth, and F. Dimroth, "4Terminal CPV Module Capable of Converting Global Normal Irradiance into Electricity," presented at the CPV-14, Puertollano, Spain, 2017.

[13] L. Li et al., "Highly-integrated Hybrid Micro-Concentrating Photovoltaics," in 2018 IEEE 7th World Conference on Photovoltaic Energy Conversion (WCPEC) (A Joint Conference of 45th IEEE PVSC, 28th PVSEC 34th EU PVSEC), 2018, pp. 1655-1657.

[14] D. Sato, et al., "Design and Evaluation of a III-V/Si Partial CPV Module for Maximization of Power Generation per Unit Module Area," IEEE J. Photovolt., vol. 9, no. 1, pp. 147-153, Jan. 2019.

[15] C. Carron, "An EPFL startup makes residential solar panels twice as efficient," 09-Jul-2016. [Online]. Available: www.webcitation.org/75XUM4JkN. 